\title{
Constraints on Airy function zeros from quantum-mechanical sum rules
}

\author{
M Belloni ${ }^{1}$ and R W Robinett ${ }^{2}$ \\ ${ }^{1}$ Physics Department, Davidson College, Davidson, NC 28035, USA \\ 2 Department of Physics, The Pennsylvania State University, University Park, PA \\ 16802, USA
}

E-mail: mabelloni@davidson.edu, rick@phys.psu.edu

\begin{abstract}
We derive new constraints on the zeros of Airy functions by using the so-called quantum bouncer system to evaluate quantum-mechanical sum rules and perform perturbation theory calculations for the Stark effect. Using commutation and completeness relations, we show how to systematically evaluate sums of the form $S_{p}(n)=\sum_{k \neq n} 1 /\left(\zeta_{k}-\zeta_{n}\right)^{p}$, for natural $p>1$, where $-\zeta_{n}$ is the $n^{t h}$ zero of $A i(\zeta)$.

PACS numbers: 02.30.Gp, 03.65.Ca, 03.65.Ge
\end{abstract}




\section{Introduction}

The study of special functions and orthogonal polynomials as solutions of some of the most important and physically relevant model problems in quantum theory has formed one of the foundations of quantum mechanics since its earliest days. Familiar examples include Hermite and Laguerre polynomials for the harmonic oscillator and Coulomb problems, spherical harmonics (Legendre polynomials) for angular momentum, Bessel functions (in two and three dimensions) for both scattering and bound-state problems, Mathieu functions for the quantum-mechanical version of the pendulum, and even trigonometric functions for the infinite square well problem.

The Airy function [1] has found many applications in classical physics, especially in optics and fluid mechanics, and as an important tool in the derivation of the WKB approximation in quantum mechanics. Airy functions are also central to the 'quantum bouncer' problem, a point mass subject to the potential

$$
V(x)= \begin{cases}F x & \text { for } x>0 \\ \infty & \text { for } x \leq 0\end{cases}
$$

This problem is amenable to a variety of approximate treatments (WKB approximation, variational methods, numerical approaches including the shooting method), all of which can be compared to the exact solutions, given in terms of the Airy function $A i(\zeta)$. This system has also received considerable recent interest as it is the simplest model for the "quantum states of neutrons in the Earth's gravitational field" observed by Nesvizhevsky et al. [2].

Gea-Banacloche [3] investigated the time-dependence of localized wave packets in such a potential, motivated by the possible application to ultra-cold atoms dropped onto an 'atomic mirror,' and demonstrated the existence of quantum wave packet revivals [4] which have been observed in a wide variety of quantum-mechanical systems. He also found for the first time numerical evidence for expressions for the normalization of the 'quantum bouncer' energy eigenstates and dipole matrix elements, $\langle n|x| k\rangle$, all of which were quickly shown to be analytically correct by Vallée [5] using earlier published work from the mathematical [6] and scientific literature [7] on Airy functions. Goodmanson [8] extended these results to produce closed-form expressions for the matrix elements of arbitrary powers of position, $\left\langle n\left|x^{q}\right| k\right\rangle$, using recursion relations. The lowest-order (dipole and quadrupole) off-diagonal elements, $\left\langle n\left|x^{1,2}\right| k\right\rangle$, have very simple forms and were found to depend on inverse powers of the combination $\left(\zeta_{n}-\zeta_{k}\right)$ where $-\zeta_{n}$ is the $n^{\text {th }}$ zero of $A i(\zeta)$.

Such differences are intrinsically related to the corresponding differences in energy eigenvalues for the stationary states, $E_{n}-E_{k}$, since for the 'quantum bouncer,' the quantized energy eigenvalues are given directly by

$$
E_{n}=\zeta_{n} \mathcal{E}_{0} \quad \text { where } \quad \mathcal{E}_{0}=\left(\frac{\hbar^{2} F^{2}}{2 m}\right)^{1 / 3} .
$$

The association of such energy differences in conjunction with dipole (and higher) matrix 
elements for a quantum-mechanical system is most familiar from energy-weighted sum rules, such as the Thomas-Reiche-Kuhn (TRK) formula [9]

$$
\sum_{k}\left(E_{k}-E_{n}\right)|\langle n|x| k\rangle|^{2}=\frac{\hbar^{2}}{2 m} \quad \text { (TRK sum rule) } .
$$

Because of the simple nature of the expressions found in Refs. [3] - [8], such sum rules automatically provide constraints on sums of inverse powers of differences of the zeros of Airy functions, and a systematic study of such relationships is the topic which we will examine here. (We note that Sukumar [10], [11] has found constraints on combinations of inverse powers of energy eigenvalues using Green's function techniques for several familiar model systems [10], including the linear potential involving the Airy function [11] studied here. Our approach is different, however, and we find new constraints on the $\zeta_{n}$ which have not, to our knowledge, appeared in the mathematical physics literature.)

In Section 2 we briefly review the quantum-mechanical solution of the 'quantum bouncer' problem in terms of Airy functions, and use a number of well-known quantum sum rules to find new constraints on Airy function zeros. Specifically, we find closed form expressions for the quantity

$$
S_{p}(n) \equiv \sum_{k \neq n} \frac{1}{\left(\zeta_{k}-\zeta_{n}\right)^{p}}
$$

for numerous natural (positive integer) values of $p$. It has also been noted that energydifference weighted sum rules have a mathematical structure similar to second-order perturbation theory [12] and we will also use results for the second-order Stark shift (the energy change due to the addition of an external constant field) in the same way. In Section 3 we then show how to systematically derive closed form expressions for the $S_{p}(n)$ in Eqn. (4) using commutation relations and closure methods (inserting a complete set of states) for all natural values of $p>1$, and exhibit specific results for $p=2, \ldots, 11$ as examples. We also discuss, in Section 4, the relationship of such results to constraints arising from other quantum-mechanical expressions, such as the famous Bethe sum rule [18] and other interconnections between the $S_{p}(n)$. Finally, in Sec. [5, we briefly discuss multi-summation expressions, generalizing Eqn. (44), motivated by constraints arising from higher orders of perturbation theory.

\section{The quantum bouncer and sum rule constraints}

The Schrödinger equation for the potential in Eqn. (11) is

$$
-\frac{\hbar^{2}}{2 m} \frac{d^{2} \psi_{n}(x)}{d x^{2}}+F x \psi_{n}(x)=E_{n} \psi_{n}(x) \quad \text { for } 0 \leq x<\infty
$$

where $\psi_{n}(x)$ and $E_{n}$ are the energy eigenfunctions and eigenvalues, respectively. The appropriate boundary conditions are $\psi_{n}(x=0)=0$ and $\psi_{n}(x \rightarrow \infty)=0$. A change of variable, $x=\rho \zeta$, transforms Eqn. (5) into

$$
\frac{d^{2} \psi_{n}(\zeta)}{d \zeta^{2}}=\left(\zeta-\bar{\zeta}_{n}\right) \psi_{n}(\zeta)
$$


where

$$
\rho=\left(\frac{\hbar^{2}}{2 m F}\right)^{1 / 3} \quad \text { and } \quad E_{n} \equiv\left(\frac{\hbar^{2} F^{2}}{2 m}\right)^{1 / 3} \bar{\zeta}_{n}=\mathcal{E}_{0} \bar{\zeta}_{n} .
$$

The solutions of Eqn. ([6) are Airy functions, namely $A i\left(\zeta-\zeta_{n}\right)$ and $B i\left(\zeta-\zeta_{n}\right)$, and only the $A i(\zeta)$ solution is acceptable since the $\psi_{n}(x)$ must be square integrable over the range $(0, \infty)$. The boundary condition at the infinite wall $(\zeta=0)$ imposes the additional constraint that $A i\left(-\bar{\zeta}_{n}\right)=0$, so that the $-\bar{\zeta}_{n}$ are simply the zeros of the relevant Airy function, $-\zeta_{n}$. Since $\bar{\zeta}_{n}=\zeta_{n}$, the energy eigenvalues are then directly given by $E_{n}=\zeta_{n} \mathcal{E}_{0}$ and familiar WKB arguments or handbook results [13] can be used to derive an approximate formula for $\zeta_{n}$ for large $n$ (quantum number), namely

$$
\zeta_{n} \sim\left[\frac{3 \pi}{2}(n-1 / 4)\right]^{2 / 3}
$$

The normalization of these states, found first numerically in Ref. [3], and then confirmed analytically in Refs. [5] and [8], make use of earlier published results (Refs. [6] and [7]), and can be written in the form

$$
\psi_{n}\left(\frac{x}{\rho}\right)=\psi_{n}(\zeta)=\frac{A i\left(\zeta-\zeta_{n}\right)}{\sqrt{\rho}\left|A i^{\prime}\left(-\zeta_{n}\right)\right|}
$$

if we include the proper dimensional constant.

Goodmanson [8] has found a recursion relationship for the position-matrix elements, which when written in terms of the normalized eigenstates and the scaled variable $\zeta=x / \rho$, reads

$$
\begin{aligned}
2 \delta_{1, p}(-1)^{n-k+1}= & p(p-1)(p-2)(p-3)\left\langle n\left|\zeta^{p-4}\right| k\right\rangle+4 p(p-1) \zeta_{\text {ave }}\left\langle n\left|\zeta^{p-2}\right| k\right\rangle \\
& -2 p(2 p-1)\left\langle n\left|\zeta^{p-1}\right| k\right\rangle+\left(\zeta_{n}-\zeta_{k}\right)^{2}\left\langle n\left|\zeta^{p}\right| k\right\rangle
\end{aligned}
$$

where $\zeta_{\text {ave }} \equiv\left(\zeta_{n}+\zeta_{k}\right) / 2$. It is understood that for a given value of $p$, any expectation values of negative powers of $\zeta$ are to be ignored.

Using this algorithm, one can find the first few diagonal and off-diagonal matrix elements needed for various sum rule calculations. For example, one has

$$
\begin{array}{ll}
p=2: & \langle n|\zeta| n\rangle=\frac{2 \zeta_{n}}{3} \\
p=3: & \left\langle n\left|\zeta^{2}\right| n\right\rangle=\frac{8 \zeta_{n}^{2}}{15} \\
p=4: & \left\langle n\left|\zeta^{3}\right| n\right\rangle=\frac{16 \zeta_{n}^{3}}{35}+\frac{3}{7} \\
p=5: & \left\langle n\left|\zeta^{4}\right| n\right\rangle=\frac{128 \zeta_{n}^{4}}{315}+\frac{80 \zeta_{n}}{63} \\
p=6: & \left\langle n\left|\zeta^{5}\right| n\right\rangle=\frac{256 \zeta_{n}^{5}}{693}+\frac{1808 \zeta_{n}^{2}}{3003}
\end{array}
$$

and

$$
p=1: \quad\langle n|\zeta| k\rangle=\frac{2(-1)^{n-k+1}}{\left(\zeta_{k}-\zeta_{n}\right)^{2}}
$$


Airy function zeros and quantum mechanics

$$
\begin{array}{ll}
p=2: & \left\langle n\left|\zeta^{2}\right| k\right\rangle=\frac{24(-1)^{n-k+1}}{\left(\zeta_{k}-\zeta_{n}\right)^{4}} \\
p=3: & \left\langle n\left|\zeta^{3}\right| k\right\rangle=(-1)^{n-k+1}\left[\frac{720}{\left(\zeta_{k}-\zeta_{n}\right)^{6}}-\frac{48 \zeta_{n}}{\left(\zeta_{k}-\zeta_{n}\right)^{4}}-\frac{24}{\left(\zeta_{k}-\zeta_{n}\right)^{3}}\right] \\
p=4: & \left\langle n\left|\zeta^{4}\right| k\right\rangle=(-1)^{n-k+1}\left[\frac{40340}{\left(\zeta_{k}-\zeta_{n}\right)^{8}}-\frac{3840 \zeta_{n}}{\left(\zeta_{k}-\zeta_{n}\right)^{6}}-\frac{1920}{\left(\zeta_{k}-\zeta_{n}\right)^{5}}\right] .
\end{array}
$$

(We can then insert the appropriate powers of $\rho$ as needed for dimensional correctness in physical matrix elements.)

We note that Eqn. (11) is consistent with the quantum-mechanical virial theorem as it gives

$$
\langle n|V(x)| n\rangle=F \rho\langle n|\zeta| n\rangle=\mathcal{E}_{0}\left(\frac{2 \zeta_{n}}{3}\right)=\frac{2}{3} E_{n}
$$

which is known to be appropriate for a symmetric power-law potential, $V_{k}(x)=V_{0}|x / a|^{k}$, with $k=1$. Other physically useful matrix elements, such as those involving $\hat{p}^{2}$, can be obtained by writing $\hat{p}^{2}=2 m(\hat{H}-F x)$. For example, this allows for the evaluation of diagonal matrix elements such as

$$
\left\langle n\left|\hat{p}^{2}\right| n\right\rangle=2 m \mathcal{E}_{0}\left(\frac{\zeta_{n}}{3}\right) \quad \text { and } \quad\left\langle n\left|\hat{p}^{4}\right| n\right\rangle=\left(2 m \mathcal{E}_{0}\right)^{2}\left(\frac{\zeta_{n}^{2}}{5}\right)
$$

or off-diagonal ones such as

$$
\left\langle n\left|\hat{p}^{2}\right| k\right\rangle=-2 m F \rho\langle n|\zeta| k\rangle=-\frac{4 \mathcal{E}_{0}(-1)^{n-k+1}}{\left(\zeta_{k}-\zeta_{n}\right)^{2}} .
$$

Using these results, we can begin cataloging the constraints which result from the application of various well-known quantum-mechanical sum rules. For example, using the energy differences $E_{k}-E_{n}=\mathcal{E}_{0}\left(\zeta_{k}-\zeta_{n}\right)$ and the off-diagonal dipole matrix elements in Eqn. (16), we start with the most-cited sum rule, the one formulated by ThomasReiche-Kuhn as in Eqn. (3). We find that all of the dimensional parameters cancel, leaving the simplest constraint we encounter, namely

$$
S_{3}(n) \equiv \sum_{k \neq n} \frac{1}{\left(\zeta_{k}-\zeta_{n}\right)^{3}}=\frac{1}{4} .
$$

This example and all of the other constraint equations for the $S_{p}(n)$ derived here, as well as the on-diagonal and off-diagonal matrix elements in Eqns. (11) - (19), can be verified to essentially arbitrary accuracy by using the numerical ability of mathematical manipulation programs such as Mathematica ${ }^{\circledR}$.

The so-called 'monopole sum rule,' which has been used in applications to nuclear collective excitations [14], is given by

$$
\sum_{k}\left(E_{k}-E_{n}\right)\left|\left\langle n\left|x^{2}\right| k\right\rangle\right|^{2}=\frac{2 \hbar^{2}}{m}\left\langle n\left|x^{2}\right| n\right\rangle,
$$

and is of a similar form to the TRK sum rule. Using the $\zeta^{2}$ matrix elements from Eqns. (12) and (17), we find the constraint equation

$$
S_{7}(n) \equiv \sum_{k \neq n} \frac{1}{\left(\zeta_{k}-\zeta_{n}\right)^{7}}=\frac{\zeta_{n}^{2}}{270} .
$$


We note that the monopole sum rule in Eqn. (24) is a special case of a more general form derived by Wang [15],

$$
\sum_{k}\left(E_{k}-E_{n}\right)|\langle n|F(x)| k\rangle|^{2}=\frac{\hbar^{2}}{2 m}\left\langle n\left|\frac{d F(x)}{d x} \frac{d F^{*}(x)}{d x}\right| n\right\rangle
$$

which simplifies if the function is real so that $F(x)=F^{*}(x)$. This general result can also be used to immediately reproduce the TRK sum rule by using $F(x)=x$.

Bethe and Jackiw [16, 17] derive several other sum rules for dipole moment matrix elements by using multiple commutation relations with the Hamiltonian, thus yielding higher powers of the energy difference. These higher-order sum rules include:

$$
\begin{aligned}
& \sum_{k}\left(E_{k}-E_{n}\right)^{2}|\langle n|x| k\rangle|^{2}=\frac{\hbar^{2}}{m^{2}}\left\langle n\left|\hat{p}^{2}\right| n\right\rangle=\frac{2 \hbar^{2}}{m}\left[E_{n}-\langle n|V(x)| n\rangle\right] \\
& \sum_{k}\left(E_{k}-E_{n}\right)^{3}|\langle n|x| k\rangle|^{2}=\frac{\hbar^{4}}{2 m^{2}}\left\langle n\left|\frac{d^{2} V(x)}{d x^{2}}\right| n\right\rangle
\end{aligned}
$$

and

$$
\sum_{k}\left(E_{k}-E_{n}\right)^{4}|\langle n|x| k\rangle|^{2}=\frac{\hbar^{4}}{m^{2}}\left\langle n\left|\left(\frac{d V(x)}{d x}\right)^{2}\right| n\right\rangle
$$

where Eqns. (28) and (29) are sometimes called the "force times momentum" and "force squared" sum rules, respectively. We recall that not all such sum rules are guaranteed to lead to convergent expressions.

The first of these three higher-order sum rules, Eqn. (27), gives the relation

$$
S_{2}=\sum_{k \neq n} \frac{1}{\left(\zeta_{k}-\zeta_{n}\right)^{2}}=\frac{\zeta_{n}}{3}
$$

This sum rule leads to a convergent result since, for large $k$, the terms in the summation scale as $1 / k^{4 / 3}$. The sum rule results in Eqns. (28) and (29), however, do not converge due to the derivatives of the discontinuous potential energy function in Eqn. (1). This implies that $S_{1}(n)$ is not convergent.

As mentioned above, it has been emphasized that the standard expression for the second-order energy shift in perturbation theory due to an added potential energy term of the form $\bar{V}(x)$, given by

$$
E_{n}^{(2)}=\sum_{k \neq n} \frac{|\langle n|\bar{V}(x)| k\rangle|^{2}}{E_{n}^{(0)}-E_{k}^{(0)}}
$$

is also a form of energy-weighted sum rule. The authors of Ref. [12] have used this fact to evaluate the Stark shift, that is the second order energy shift due to an external constant field, with a potential of the form $\bar{V}(x)=\bar{F} x$, in two model systems, the infinite square well and single attractive $\delta$-function potential. Then, using the same mathematical techniques as for the confirmation of many other sum rules in those two cases (where the relevant tools are the Mittag-Lefler theorem and standard contour integration methods respectively) one can evaluate $E_{n}^{(2)}$ in closed form. 
In this situation, the addition of a uniform external field $\bar{V}(x)=\bar{F} x$ to the potential in Eqn. (11) leads to a soluble problem with a simple redefinition of the constant force, $F \rightarrow F+\bar{F}$, giving an exact value for the new energy eigenvalues,

$$
\tilde{E}_{n}=\zeta_{n}\left[\frac{\hbar^{2}(F+\bar{F})^{2}}{2 m}\right]^{1 / 3}=E_{n}\left[1+\frac{\bar{F}}{F}\right]^{2 / 3} \quad \text { where } \quad E_{n}=\mathcal{E}_{0} \zeta_{n} .
$$

The term in brackets can be easily expanded giving predictions for the first-, second-, and third-order perturbation theory results, namely

$$
E_{n}^{(1)}=\frac{2}{3}\left(\frac{\bar{F}}{F}\right)\left(\mathcal{E}_{0} \zeta_{n}\right), \quad E_{n}^{(2)}=-\frac{1}{9}\left(\frac{\bar{F}}{F}\right)^{2}\left(\mathcal{E}_{0} \zeta_{n}\right), \quad E_{n}^{(3)}=\frac{4}{81}\left(\frac{\bar{F}}{F}\right)^{3}\left(\mathcal{E}_{0} \zeta_{n}\right) .
$$

The first-order result is easily confirmed by noting that

$$
E_{n}^{(1)}=\langle n|\bar{F} x| n\rangle=\bar{F} \rho\langle n|\zeta| n\rangle=\left(\frac{\bar{F}}{F}\right)(F \rho)\left(\frac{2 \zeta_{n}}{3}\right)=\frac{2}{3}\left(\frac{\bar{F}}{F}\right)\left(\mathcal{E}_{0} \zeta_{n}\right)
$$

where we use the diagonal dipole matrix element in Eqn. (11). The second-order shift equation can then be used as a new constraint on a different combination of Airy function zeros, giving

$$
S_{5}(n)=\sum_{k \neq n} \frac{1}{\left(\zeta_{k}-\zeta_{n}\right)^{5}}=\frac{\zeta_{n}}{36}
$$

and we note that $S_{2}(n)=12 S_{5}(n)$. Higher-order perturbative corrections can in principle be used to derive closed form expressions for more complex combinations of inverse powers of $\left(\zeta_{k}-\zeta_{n}\right)$, since one can expand the exact result in Eqn. (32) to arbitrarily high order. In fact, we use the third-order expression to briefly discuss multi-index summation generalizations of Eqn. (4) in Sec. 5 ,

To briefly summarize, the evaluation of several well-known quantum-mechanical sum rules and the evaluation of the related energy-weighted sum over dipole matrix elements from perturbation theory have provided closed form expressions for $S_{p}(n)$ for $p=2,3,5$ and 7 . In the next section, we show how to systematically evaluate $S_{p}(n)$ for all natural $p>1$.

\section{Systematic method for construction of $S_{p}(n)$}

Before proceeding, we recall the techniques that are used in the derivation of many of the familiar quantum-mechanical sum rules, especially those involving dipole matrix elements. As an example, for the TRK sum rule in Eqn. (3) , we write the standard $x, \hat{p}$ commutation relation, $[\hat{p}, x]=-i \hbar$, bracketed by energy eigenstates:

$$
-i \hbar=\langle n|\hat{p} x-x \hat{p}| n\rangle=\sum_{\text {all } k}\{\langle n|\hat{p}| k\rangle\langle k|x| n\rangle-\langle n|x| k\rangle\langle k|\hat{p}| n\rangle\}
$$

where we have also inserted a complete set of states to obtain the right-hand side of the equality. We can then use a second commutation relation, namely

$$
[\hat{H}, x]=\frac{1}{2 m}\left[\hat{p}^{2}, x\right]=\frac{\hbar}{m i} \hat{p},
$$


where we assume a standard one-dimensional Hamiltonian of the form $\hat{H}=\hat{p}^{2} / 2 m+$ $V(x)$, to write

$$
\langle n|\hat{p}| k\rangle=\frac{i m}{\hbar}\langle n|[\hat{H}, x]| k\rangle=\frac{i m\left(E_{n}-E_{k}\right)}{\hbar}\langle n|x| k\rangle .
$$

There is a similar expression for $\langle k|\hat{p}| n\rangle$ and combining these two results in Eqn. (36) gives the TRK sum rule. Note that Eqn. (38) gives $\langle n|\hat{p}| k\rangle=0$ if $n=k$ as is appropriate for energy eigenstates where the average momentum should vanish in a stationary state.

Specializing now to the case of the quantum bouncer, and using the result in Eqn. (16), we find the more specific result,

$$
\langle n|\hat{p}| k\rangle=\frac{\hbar}{i \rho} \frac{(-1)^{n-k+1}}{\left(\zeta_{k}-\zeta_{n}\right)}
$$

and we note that $\langle n|\hat{p}| k\rangle^{*}=\langle k|\hat{p}| n\rangle$. This is the lowest-order (in inverse powers of $\left.\zeta_{k}-\zeta_{n}\right)$ term possible, and is our starting point.

We see that the important ingredients are insertion of a complete set of states, the matrix-element connection in Eqn. (38), and appropriate commutation relations. Motivated by these methods, we start with the simple closure relationship for the momentum operator, namely

$$
\sum_{\text {all } k}\langle n|\hat{p}| k\rangle\langle k|\hat{p}| n\rangle=\left\langle n\left|\hat{p}^{2}\right| n\right\rangle .
$$

The only non-zero elements on the left-hand side are the off-diagonal matrix elements in Eqn. (39), while the diagonal matrix element on the right-hand side can be evaluated using Eqn (21). Inserting these results gives

$$
S_{2}(n)=\frac{\zeta_{n}}{3}
$$

This is the same result as obtained from the sum rule in Eqn. (27), which is correct since that expression is most simply obtained from the closure relationship in Eqn. (40), using the expression in Eqn. (38) twice.

The next higher power of $\left(\zeta_{k}-\zeta_{n}\right)^{-1}$ is obtained by inserting a complete of states into the commutation relation $[x, \hat{p}]=i \hbar$, namely

$$
\sum_{\text {all } k}\{\langle n|x| k\rangle\langle k|\hat{p}| n\rangle-\langle n|\hat{p}| k\rangle\langle k|x| n\rangle\}=\langle n|i \hbar| n\rangle=i \hbar
$$

which, of course, reproduces the TRK sum rule result, giving $S_{3}(n)=1 / 4$. We note here that in any summation involving $\langle n|\hat{p}| k\rangle$, the $n=k$ term is not present.

To evaluate $S_{4}(n)$, we use the $x$-closure relationship,

$$
\sum_{\text {all } k}\langle n|x| k\rangle\langle k|x| n\rangle=\left\langle n\left|x^{2}\right| n\right\rangle
$$

and by explicitly including both on- and off-diagonal terms on the left-hand side (and removing all dimensional constants) this gives us

$$
|\langle n|\zeta| n\rangle|^{2}+\sum_{k \neq n} \frac{4}{\left(\zeta_{k}-\zeta_{n}\right)^{4}}=\left\langle n\left|\zeta^{2}\right| n\right\rangle
$$


or

$$
\left(\frac{2 \zeta_{n}}{3}\right)^{2}+4 S_{4}(n)=\frac{8 \zeta_{n}^{2}}{15} \quad \text { yielding } \quad S_{4}(n)=\frac{\zeta_{n}^{2}}{45} .
$$

The correct iterative procedure required to evaluate $S_{p}(n)$ for any natural value of $p$ is now clear. The off-diagonal matrix elements for $\left\langle n\left|\zeta^{q}\right| k\right\rangle$ will have a leading term of order $\left(\zeta_{k}-\zeta_{n}\right)^{-2 q}$. We then apply closure to the general commutator result $\left[x^{q}, \hat{p}\right]=i q \hbar x^{q-1}$ in the form

$$
\sum_{\text {all } k}\left\{\left\langle n\left|x^{q}\right| k\right\rangle\langle k|\hat{p}| n\rangle-\langle n|\hat{p}| k\rangle\left\langle k\left|x^{q}\right| n\right\rangle\right\}=i q \hbar\left\langle n\left|x^{q-1}\right| n\right\rangle .
$$

The recursive relation in Eqn. (10) can then be used straightforwardly (if tediously) to evaluate both $\left\langle n\left|x^{q}\right| k\right\rangle$ and $\left\langle n\left|x^{q-1}\right| n\right\rangle$ to obtain a closed-form expression for $S_{2 q+1}(n)$ in terms of explicit powers of $\zeta_{n}$ and values of $S_{p}(n)$ for $p<2 q$. This procedures allows one to increment the value of $p$ by one, since the inclusion of the $\langle n|\hat{p}| k\rangle$ matrix elements adds one more inverse power of $\left(\zeta_{k}-\zeta_{n}\right)$.

To evaluate $S_{p}(n)$ for $p$ values two units higher, we generalize the $x$-closure relationship in Eqn. (43) to

$$
\sum_{\text {all } k}\left\langle n\left|x^{q}\right| k\right\rangle\langle k|x| n\rangle=\left\langle n\left|x^{q+1}\right| n\right\rangle
$$

and again evaluation of only $x$-dependent on- and off-diagonal matrix elements will suffice to obtain an expression for $S_{2 q+2}(n)$, as the $\langle k|x| n\rangle$ term adds two inverse powers of $\left(\zeta_{k}-\zeta_{n}\right)$.

In this way, we are able to systematically evaluate $S_{p}(n)$ for all $p>1$, since the $p=1$ case, corresponding to use of Eqn. (28), does not converge. We present below results obtained in this way for the cases $p=2, \ldots, 11$,

$$
\begin{aligned}
& S_{2}(n)=\frac{\zeta_{n}}{3} \\
& S_{3}(n)=\frac{1}{4} \\
& S_{4}(n)=\frac{\zeta_{n}^{2}}{45} \\
& S_{5}(n)=\frac{\zeta_{n}}{36} \\
& S_{6}(n)=\frac{2 \zeta_{n}^{3}}{945}+\frac{1}{112} \\
& S_{7}(n)=\frac{\zeta_{n}^{2}}{270} \\
& S_{8}(n)=\frac{\zeta_{n}^{4}}{4725}+\frac{5 \zeta_{n}}{2268} \\
& S_{9}(n)=\frac{\zeta_{n}^{3}}{2100}+\frac{1}{2240} \\
& S_{10}(n)=\frac{2 \zeta_{n}^{5}}{93555}+\frac{611 \zeta_{n}^{2}}{1496880} \\
& S_{11}(n)=\frac{\zeta_{n}^{4}}{17010}+\frac{43 \zeta_{n}}{272160},
\end{aligned}
$$


all of which have also been verified numerically.

\section{Other sum rule constraints}

There are a large number of other possible constraints, obtainable from both the use of the systematic approach developed above, or through other familiar sum rules.

For increasing values of $p$, there are often several closure-motivated constraints that can be applied to obtain closed-form expressions for $S_{p}(n)$. For example, to evaluate $S_{8}(n)$, we can use either

$$
\sum_{\text {all } k}\left\langle n\left|x^{3}\right| k\right\rangle\langle k|x| n\rangle=\left\langle n\left|x^{4}\right| n\right\rangle=\sum_{\text {all } k}\left\langle n\left|x^{2}\right| k\right\rangle\left\langle k\left|x^{2}\right| n\right\rangle
$$

and use one as a cross-check against the other. Alternatively, such identities can be used as further constraints among the various $S_{p}(n)$. The expression above, for example, requires that

$$
\begin{aligned}
& \left(\frac{16 \zeta_{n}^{3}}{35}+\frac{3}{7}\right)\left(\frac{2 \zeta_{n}}{3}\right)+1440 S_{8}(n)-96 \zeta_{n} S_{6}(n)-47 S_{5}(n) \\
& =\left(\frac{8 \zeta_{n}^{2}}{15}\right)^{2}+576 S_{8}(n)
\end{aligned}
$$

which is easily verified.

One of the more famous constraints on dipole matrix elements is the Bethe sum rule [18]

$$
\sum_{k}\left(E_{k}-E_{n}\right)\left|\left\langle n\left|e^{i q x}\right| k\right\rangle\right|^{2}=\frac{\hbar^{2} q^{2}}{2 m},
$$

which was developed in the study of energy loss mechanisms, eventually leading to the Bethe-Bloch formula. This single sum rule actually provides an infinite tower of constraints on the $S_{p}(n)$. To see this, we expand the matrix element of the exponentials via

$$
\left\langle n\left|e^{i q x}\right| k\right\rangle=\langle n \mid k\rangle+i q\langle n|x| k\rangle-\frac{q^{2}}{2 !}\left\langle n\left|x^{2}\right| k\right\rangle-i \frac{q^{3}}{3 !}\left\langle n\left|x^{3}\right| k\right\rangle+\frac{q^{4}}{4 !}\left\langle n\left|x^{4}\right| k\right\rangle+\cdots
$$

The odd-order (in $q$ ) terms on the left-hand side of Eqn. (60) automatically cancel since it is an even function by construction, while the $\mathcal{O}\left(q^{0}\right)$ terms is absent since $\langle n \mid k\rangle=0$ if $n \neq k$. The lowest non-vanishing term, the $\mathcal{O}\left(q^{2}\right)$ term on the left-hand side, saturates the right-hand side by reproducing the TRK sum rule. All higher-order terms must therefore vanish. For example, the vanishing of the $\mathcal{O}\left(q^{4}\right)$ term implies that

$$
\frac{1}{4} \sum_{k}\left(E_{k}-E_{n}\right)\left\langle n\left|x^{2}\right| k\right\rangle\left\langle k\left|x^{2}\right| n\right\rangle=\frac{1}{3} \sum_{k}\left(E_{k}-E_{n}\right)\left\langle n\left|x^{3}\right| k\right\rangle\langle k|x| n\rangle
$$

which is a different constraint equation involving $S_{8}(n)$ and including lower-order terms than the one in Eqn. (58). 
Closure relationships involving mixed combinations of $x$ and $\hat{p}$ of higher order in the momentum operator are also possible, often reproducing earlier results. For example, the closure-relation for $\hat{p}^{4}$, namely

$$
\sum_{\text {all } k}\left\langle n\left|\hat{p}^{2}\right| k\right\rangle\left\langle k\left|\hat{p}^{2}\right| n\right\rangle=\left\langle n\left|\hat{p}^{4}\right| n\right\rangle
$$

can be used in conjunction with Eqns. (21) and (22) to evaluate $S_{4}(n)$. Wang [15] has also derived a number of sum rules involving mixed position- and momentum-matrix elements which can be used.

\section{Multi-index summations}

To explore more complex relationships involving inverse powers of $\left(\zeta_{k}-\zeta_{n}\right)$, we extend the perturbation theory analysis of the Stark effect for the quantum bouncer to third order. We first recall that the third-order correction in perturbation theory due to a general $\bar{V}(x)$ term is given by

$$
\begin{aligned}
E_{n}^{(3)}= & \sum_{k \neq n} \sum_{j \neq n} \frac{\langle n|\bar{V}(x)| k\rangle\langle k|\bar{V}(x)| j\rangle\langle j|\bar{V}(x)| n\rangle}{\left(E_{n}^{(0)}-E_{k}^{(0)}\right)\left(E_{n}^{(0)}-E_{j}^{(0)}\right)} \\
& -\langle n|\bar{V}(x)| n\rangle \sum_{k \neq n} \frac{|\langle n|\bar{V}(x)| k\rangle|^{2}}{\left(E_{n}^{(0)}-E_{k}^{(0)}\right)^{2}} .
\end{aligned}
$$

Using $\bar{V}=\bar{F} x$ as above, the exact third-order result in Eqn. (33), and removing all dimensional factors gives the constraint

$$
\begin{aligned}
\frac{4 \zeta_{n}}{81}=\sum_{k \neq j \neq n} & \frac{\langle n|\zeta| k\rangle\langle k|\zeta| j\rangle\langle j|\zeta| n\rangle}{\left(\zeta_{k}-\zeta_{n}\right)\left(\zeta_{j}-\zeta_{n}\right)}+\sum_{j=k \neq n} \frac{|\langle n|\zeta| k\rangle|^{2}\langle k|\zeta| k\rangle}{\left(\zeta_{k}-\zeta_{n}\right)^{2}} \\
& -\langle n|\zeta| n\rangle \sum_{k \neq n} \frac{|\langle n|\zeta| k\rangle|^{2}}{\left(\zeta_{k}-\zeta_{n}\right)^{2}} .
\end{aligned}
$$

In evaluating the double sum, we have separated off the cases where $j=k \neq n$, leaving the distinct $j \neq k \neq n$ terms.

Inserting the appropriate diagonal and off-diagonal matrix elements in Eqns. (11) and (16), we then find the relationship

$$
-8 \sum_{k \neq j \neq n} \frac{1}{\left(\zeta_{k}-\zeta_{n}\right)^{3}\left(\zeta_{k}-\zeta_{j}\right)^{2}\left(\zeta_{j}-\zeta_{n}\right)^{3}}+\frac{8}{3}\left[S_{5}(n)+\zeta_{n} S_{6}(n)\right]-\frac{8 \zeta_{n}}{3} S_{6}(n)=\frac{4 \zeta_{n}}{81}
$$

Or

$$
T_{3,2,3}(n) \equiv \sum_{k \neq j \neq n} \frac{1}{\left(\zeta_{k}-\zeta_{n}\right)^{3}\left(\zeta_{k}-\zeta_{j}\right)^{2}\left(\zeta_{j}-\zeta_{n}\right)^{3}}=\frac{\zeta_{n}}{324},
$$

a remarkably simple identity, which we have also confirmed numerically.

The presence of double (or higher) summations can be systematized, as in Sec. 3, by introducing more than one insertion of a complete set of states into any quantum identity, either resulting from simple closure or commutation relations. For example, we can write

$$
\sum_{\text {all } k \text { all } j}\langle n|x| k\rangle\langle k|x| j\rangle\langle j|x| n\rangle=\left\langle n\left|x^{3}\right| n\right\rangle .
$$


In evaluating the double sum, we must consider the following special cases, namely (i) $n=k=j$, (ii) two equal contributions arising from $n=k \neq j$ and $n=j \neq k$, (iii) $j=k \neq n$, and (iv) the completely distinct double sum where $j \neq k \neq n$. Including all of these possibilities gives the constraint

$$
\begin{aligned}
& \left(\frac{2 \zeta_{n}}{3}\right)^{3}+2\left(\frac{8 \zeta_{n}}{3} S_{4}(n)\right)+\frac{8}{3}\left[S_{3}(n)+\zeta_{n} S_{4}(n)\right] \\
& -8 \sum_{j \neq k \neq n} \frac{1}{\left(\zeta_{k}-\zeta_{n}\right)^{2}\left(\zeta_{j}-\zeta_{k}\right)^{2}\left(\zeta_{j}-\zeta_{n}\right)^{2}}=\frac{16 \zeta_{n}^{3}}{35}+\frac{3}{7}
\end{aligned}
$$

or

$$
T_{2,2,2}=\frac{2 \zeta_{n}^{3}}{945}+\frac{5}{168}
$$

which we have also confirmed numerically. Clearly an infinite number of multi-index summations can be evaluated in closed form in this way.

\section{Conclusions and future directions}

In conclusion, motivated by identities derived using a variety of quantum-mechanical sum rules, we have developed techniques to systematically evaluate sums of the form in Eqn. (4) for arbitrary natural $p>1$, explicitly exhibiting results for $S_{p}(n)$ for $p=2, \ldots, 11$. In addition, we have identified many other additional constraints on the $S_{p}(n)$ arising from self-consistency of the method and the Bethe sum rule. Using higher-order perturbation theory results as a starting point, we have also discussed the existence of multi-summation constraints arising from the the repeated use of insertion of a complete set of states. Future work might involve attempts at inductively generated closed form expressions for the $S_{p}(n)$ as well as exploration of the algebraic structures suggested by the pattern of results in Eqns. (48) - (57), where each term only contains powers of $\zeta_{n}$ modulo three.

\section{References}

[1] Vallée O and Soares M 2004 Airy Functions and Applications to Physics (New Jersey: World Scientific)

[2] Nesvizhevsky V V et al. 2002 Quantum states of neutrons in the Earth's gravitational field Nature 415 297-9; Measurement of quantum states of neutrons in the Earth's gravitational field Phys. Rev. D 67102002 (9 pages)

[3] Gea-Banacloche J 1999 A quantum bouncing ball Am. J. Phys. 67 776-782

[4] Robinett R W 2004 Quantum wave packet revivals Phys. Rep. 392 1-119

[5] Vallée O 2000 Comment on 'A quantum bouncing ball' by Julio Gea-Banacloche Am. J. Phys. 68 $672-673$

[6] Albright J R 1977 Integrals of products of Airy functions J. Phys. A: Math. Gen. 10 485-490

[7] Gordon R G 1969 New method for constructing wavefunctions for bound states and scattering $J$. Chem. Phys. 51 14-25 Appendix B

[8] Goodmanson D M 2000 A recursion relation for matrix elements of the quantum bouncer. Comment on 'A quantum bouncing ball' by Julio Gea-Banacloche Am. J. Phys. 68 866-868 
[9] Thomas W 1925 Über die Zahl der Dispersionselectronen, die einem starionären Zustande zugeordnet sind (Vorläufige Mitteilung)," Naturwissenschaftern 13 627; Kuhn W 1925 Über die Gesamtstärke der von einem Zustande ausgehenden Absorptionslinien Z. Phys. 33 408-412; Reiche F and Thomas W 1925 Über die Zahl der dispersionselektronen, die einem stationären Zustand zugeordnet sind Z. Phys. 34 510-525

[10] Sukumar C V 1990 Green's functions and a hierarchy of sum rules for the eigenvalues of confining potentials Am. J. Phys. 58 561-565

[11] Sukumar C V 2006 Sum rules for confining potentials (Preprint arXiv:quant-ph/0611066 1)

[12] Belloni M and Robinett R W 2008 Quantum mechanical sum rules for two model systems Am. J. Phys. 76 798-806 (Preprint arXiv:0802.2217)

[13] Abramowitz M and Stegun I A (1965) Handbook of Mathematical Functions (Washington, D. C.:National Bureau of Standards, U. S. Printing Office) p. 450.

[14] Bohigas O, Lane A M, and Martorell J 1979 Sum rules for nuclear collective excitations Phys. Rep. 5 267-316

[15] Wang S 1999 Generalization of the Thomas-Reiche-Kuhn and the Bethe sum rules Phys. Rev. A $60262-266$

[16] Bethe H and Jackiw R (1968) Intermediate Quantum Mechanics Second edition (New York: Benjamin) Chapter 11

[17] Jackiw R 1967 Quantum mechanical sum rules Phys. Rev. 157 1220-1225

[18] Bethe H (1930) Zur Theorie des Durchgangs schneller Korpuskularstrahlen durch Materie Ann. Phys. (Leipzig) 5 325-400; translated as Theory of the passage of fast corpuscular rays through matter in Selected Works of Hans A. Bethe with Commentary H. Bethe World Scientific Series in 20th Century Physics Vol. 18 (Singapore: World Scientific) pp. 77-154 (1997). 